\title{
Unity-Rate Codes Maximize the Normalized Throughput of On-Off Keying Visible Light Communication
}

\author{
Zunaira Babar, Hung Viet Nguyen, Panagiotis Botsinis, Dimitrios Alanis, \\ Daryus Chandra, Soon Xin Ng and Lajos Hanzo
}

\begin{abstract}
In this letter, we aim for maximizing the throughput of a VLC system. Explicitly, we conceive a Soft-In Soft-Out (SISO) decoder providing soft feedback for the classic Run Length Limited (RLL) codes, hence facilitating iterative decoding for exchanging valuable extrinsic information between the RLL and the error correction modules of a VLC system. Furthermore, we propose a Unity Rate Code (URC) for our VLC system, which hence becomes capable of matching the On-Off Keying (OOK) capacity, while maintaining a flicker-free dimming value of $50 \%$.
\end{abstract}

Keywords-Visible Light Communication, Iterative Decoding, EXIT charts, concatenated RLL codes.

\section{INTRODUCTION}

Visible Light Communication (VLC) serves the dual purpose of illumination as well as communication. In this context, a VLC system should be capable of mitigating any undesirable flickering resulting from the modulation of light, while also supporting a wide range of dimming values [1]. In the simplest VLC system, relying on On-Off Keying (OOK) modulation, this is achieved by invoking Run Length Limited (RLL) line coding techniques, such as the 4B6B and 8B10B schemes [1], which yield a constant (flicker-free) dimming level of $50 \%$. The ratio of the on-time and off-time of the signal may then be adjusted to meet the arbitrary dimming level set by the user.

RLL codes typically exhibit a weak error correction capability. Consequently, a VLC system also incorporates Forward Error Correction (FEC) codes for mitigating the deleterious effects of noise. As another limitation, RLL schemes intrinsically support only hard decoding, which limits the error correction potential of the FEC codes. This issue was first addressed in [2], where a multiple-candidate-based Soft-In Hard-Out (SIHO) RLL decoder was conceived for a VLC system relying on the $4 \mathrm{~B} 6 \mathrm{~B}$ and $8 \mathrm{~B} 10 \mathrm{~B}$ techniques. The SIHO scheme of [2] was further developed into the Soft-In Soft-Out (SISO) decoding in [3], [4]. However, the designs of [3], [4] did not invoke iterative decoding between the RLL and FEC modules.

The authors are with the School of Electronics and Computer Science, University of Southampton, SO17 1BJ, United Kingdom. Email: \{zb2g10, hvn08r, pb1y14, da4g11, dc2n14, sxn, lh\}@ecs.soton.ac.uk.

The financial support of the European Research Council under the Advanced Fellow Grant, that of the Royal Society's Wolfson Research Merit Award and that of the Engineering and Physical Sciences Research Council under Grant EP/L018659/1 is gratefully acknowledged. The research data for this paper is available at [http://doi.org/10.5258/SOTON/402170].
The first joint RLL-FEC coding scheme was conceived in [5], where the 1/2-rate Biphase Mark Code $(\mathrm{BMC})^{1}$ constitutes the recursive inner component. Motivated by these developments, we propose new iterative RLL-FEC schemes, which rely on more efficient inner components for the sake of maximizing the achievable throughput, or alternatively, for improving the achievable Bit Error Ratio (BER) performance at a constant system throughput. Explicitly, we conceive a SISO decoder for the Look-Up Table (LUT) based RLL codes, such as the 4B6B, which relies on soft feedback in contrast to the SISO decoders of [3], [4]. Hence, the resultant SISO decoder is capable of exchanging extrinsic information with the FEC module using iterative decoding, thereby improving the performance. As an alternative strategy, we also propose to replace the classic $R L L$ code with a recursive Unity Rate Code (URC) for the sake of circumventing the irrecoverable capacity loss associated with the classic RLL codes. We demonstrate that the URC is capable of ensuring a dimming level of $50 \%$. We quantify the benefits of the proposed $4 B 6 B$ and URC aided VLC schemes by invoking the 17-subcode IRregular Convolutional Code (IRCC) of [6] as the outer decoding component, whose weighting coefficients are optimized using EXtrinsic information Transfer (EXIT) charts [7]. It is demonstrated that the 4B6B and URC aided $V L C$ systems designed offer higher throughput than their BMC aided counterpart.

This letter is structured as follows. In Section II, we detail our system model, followed by our proposed inner components in Section III. Our results are discussed in Section IV, while our conclusions are offered in Section V.

\section{SySTEM MODEL}

Fig. 1 shows the general schematic of a VLC system, relying on a concatenated coding scheme, where the FEC block constitutes the outer component, while the inner component is an RLL or scrambler, which ensures a $50 \%$ dimming level at the output. At the transmitter, the intended information $x_{1}$ is first encoded by the FEC encoder of Fig. 1. The FEC-encoded bits $\bar{x}_{1}$ are interleaved $(\pi)$ for generating the permuted bit sequence $x_{2}$, which is fed to an inner encoder. The resultant output $\bar{x}_{2}$ is OOK modulated into $\bar{x}(t)$ and subsequently emitted as a VLC signal. The transmitted signal is received

\footnotetext{
${ }^{1}$ The line coding technique is termed 'Miller' code in [5]. However, the trellis used in [5] is that of a 'Biphase Mark' code.
} 


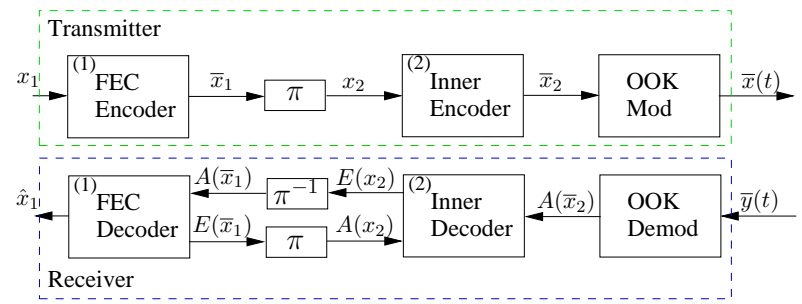

Fig. 1: Schematic of a VLC system.

by the photodetector at the destination. The detected signal $\bar{y}(t)$ is fed to the OOK demodulator ('OOK Demod') of Fig. 1, which produces the discrete signal $\bar{y}_{2}=x_{2}+n$, where $n$ is the Additive White Gaussian Noise (AWGN) having a variance of $\sigma^{2}$. Based on the demodulated output $\bar{y}_{2}$, the OOK demodulator computes the corresponding a-priori Logarithmic-Likelihood Ratios (LLRs) $A\left(\bar{x}_{2}\right)$, which are fed to the inner SISO decoder. Finally, iterative decoding is invoked for exchanging extrinsic information between the inner and outer decoders, as shown in Fig. 1, where the notations $A($. and $E($.$) denote the a-priori and extrinsic LLRs, respectively.$

The concatenated code design of Fig. 1 may be optimized with the aid of EXIT charts [7], which is a semi-analytical tool conceived for analyzing the exchange of average Mutual Information (MI) between the inner and outer decoders. Explicitly, the inner and outer decoder's EXIT curves may be constructed by modeling the associated a priori information, $A\left(x_{2}\right)$ and $A\left(\bar{x}_{1}\right)$ respectively, using an independent Gaussian distribution for a range of $I_{A}(z) \in[0,1]$, where $I_{A}(z)$ denotes the average MI between $z$ and $A(z)$, while $z \in\left\{x_{2}, \bar{x}_{1}\right\}$. The resultant extrinsic MI $I_{E}\left(x_{2}\right)$ and $I_{E}\left(\bar{x}_{1}\right)$ are then formulated for the inner and outer decoders as:

$$
I_{E}(z)=1-\mathrm{E}\left(\mathrm{H}_{b}\left(\frac{1}{1+e^{E(z)}}\right)\right),
$$

where $\mathrm{E}($.$) denotes the expectation operator (time-average),$ while $\mathrm{H}_{b}($.$) is the binary entropy function. The MI charac-$ teristics defined by $I_{A}\left(x_{2}\right)$ and $I_{E}\left(x_{2}\right)$ constitute the inner decoder's EXIT curve, while $I_{A}\left(\bar{x}_{1}\right)$ and $I_{E}\left(\bar{x}_{1}\right)$ yield the outer decoder's EXIT curve. Finally, both the inner and outer decoder's EXIT curves are plotted in the same graph, with the vertical and horizontal axis of the outer decoder swapped.

\section{PRoposed InNER COMPONENTS}

\section{A. Soft-In Soft-Out RLL Decoder Relying on Feedback}

Let us consider an LUT-based $(q, s)$ RLL scheme, which maps its $q$-bit input onto a unique $s$-bit output, so that the resultant $s$ bits have an equal number of 1's and 0's. For example, we have $q=4$ and $s=6$ for the 4B6B RLL code. Hence, the RLL encoder of Fig. 1 takes as its input $x_{2}=\left[x_{2,1}, \ldots, x_{2, i}, \ldots, x_{2, N}\right]$, where we have $x_{2, i}=\left[x_{2, i}^{(1)}, \ldots, x_{2, i}^{(j)}, \ldots, x_{2, i}^{(q)}\right]$, to yield the $s$-bit output $\bar{x}_{2}=\left[\bar{x}_{2,1}, \ldots, \bar{x}_{2, i}, \ldots, \bar{x}_{2, N}\right]$, where we have $\bar{x}_{2, i}=$ $\left[\bar{x}_{2, i}^{(1)}, \ldots, \bar{x}_{2, i}^{(j)}, \ldots, \bar{x}_{2, i}^{(s)}\right]$. Similarly, the OOK demodulated signal may be represented as $\bar{y}_{2}=\left[\bar{y}_{2,1}, \ldots, \bar{y}_{2, i}, \ldots, \bar{y}_{2, N}\right]$, where we have $\bar{y}_{2, i}=\left[\bar{y}_{2, i}^{(1)}, \ldots, \bar{y}_{2, i}^{(j)}, \ldots, \bar{y}_{2, i}^{(s)}\right]$. Based on this notation, the proposed SISO RLL decoder computes the extrinsic information $E\left(x_{2}\right)$ for feedback as follows:

1) The SISO RLL decoder gleans the a-priori information $A\left(\bar{x}_{2}\right)$ from the OOK demodulator, defined as:

$$
A\left(\bar{x}_{2, i}^{(j)}\right) \triangleq \ln \left(\frac{\mathrm{P}\left(\bar{y}_{2, i}^{(j)} \mid \bar{x}_{2, i}^{(j)}=1\right)}{\mathrm{P}\left(\bar{y}_{2, i}^{(j)} \mid \bar{x}_{2, i}^{(j)}=0\right)}\right)=\frac{1}{2 \sigma^{2}}\left(2 \bar{y}_{2, i}^{(j)}-1\right) .
$$

2) Since each bit of the $s$-bit codeword is independently transmitted over a VLC channel, the codeword-based probabilities are computed using the bit-based probabilities of Eq. (2) as follows:

$$
\mathrm{P}\left(\bar{y}_{2, i} \mid \bar{x}_{2, i}\right)=\prod_{j=1}^{s} \mathrm{P}\left(\bar{y}_{2, i}^{(j)} \mid \bar{x}_{2, i}^{(j)}\right) .
$$

Since the RLL encoder maps each $q$-bit information word onto a unique $s$-bit codeword, we have:

$$
\mathrm{P}\left(\bar{y}_{2, i} \mid \bar{x}_{2, i}\right)=\mathrm{P}\left(\bar{y}_{2, i} \mid x_{2, i}\right),
$$

where $\bar{x}_{2, i}=\mu\left(x_{2, i}\right)$ for the RLL mapper $\mu$.

3) The a-posteriori probability $\mathrm{P}\left(x_{2, i}^{(j)} \mid \bar{y}_{2, i}\right)$ may be computed using the a-priori information of Eq. (4) and the a-priori information $A\left(x_{2}\right)$ gleaned from the outer decoder, which is defined as:

$$
A\left(x_{2, i}^{(j)}\right) \triangleq \ln \left(\frac{\mathrm{P}\left(x_{2, i}^{(j)}=1\right)}{\mathrm{P}\left(x_{2, i}^{(j)}=0\right)}\right) .
$$

Explicitly, according to the Bayes' rule, we have:

$$
\begin{aligned}
\mathrm{P}\left(x_{2, i}=\chi \mid \bar{y}_{2, i}\right) & =\frac{\mathrm{P}\left(\bar{y}_{2, i} \mid x_{2, i}=\chi\right) \mathrm{P}\left(x_{2, i}=\chi\right)}{\mathrm{P}\left(\bar{y}_{2, i}\right)} \\
& \approx \mathrm{P}\left(\bar{y}_{2, i} \mid x_{2, i}=\chi\right) \mathrm{P}\left(x_{2, i}=\chi\right), \\
& =\mathrm{P}\left(\bar{y}_{2, i} \mid x_{2, i}=\chi\right) \prod_{l=1}^{q} \mathrm{P}\left(x_{2, i}^{(l)}=\chi^{(l)}\right),
\end{aligned}
$$

where $\chi=\left[\chi^{(1)}, \ldots, \chi^{(j)}, \ldots, \chi^{(q)}\right]$ is a hypothetical $q$-bit information word. The corresponding bit-based $a$ posteriori probabilities may be calculated by marginalizing the probabilities of Eq. (6) as:

$$
\mathrm{P}\left(x_{2, i}^{(j)}=b \mid \bar{y}_{2, i}\right)=\sum_{\chi \mid \chi^{(j)}=b} \mathrm{P}\left(x_{2, i}=\chi \mid \bar{y}_{2, i}\right),
$$

for $b \in\{0,1\}$. Based on Eq. (6) and Eq. (7), the SISO RLL decoder generates the extrinsic probabilities using:

$$
\begin{aligned}
& \mathrm{P}_{e}\left(x_{2, i}^{(j)}=b \mid \bar{y}_{2, i}\right)= \\
& \sum_{\chi \mid \chi^{(j)}=b}\left(\mathrm{P}\left(\bar{y}_{2, i} \mid x_{2, i}=\chi\right) \prod_{l \neq j}^{\forall l} \mathrm{P}\left(x_{2, i}^{(l)}=\chi^{(l)}\right)\right),
\end{aligned}
$$




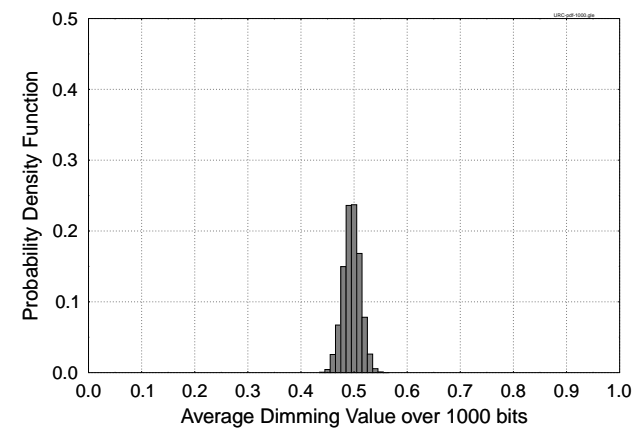

(a)

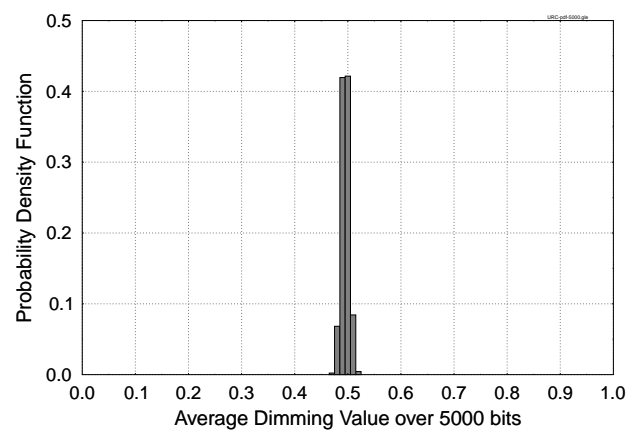

(b)

Fig. 2: Probability density function of the average dimming value at the output of the URC.

which are fed back to the outer decoder as:

$$
E\left(x_{2, i}^{(j)}\right) \triangleq \ln \left(\frac{\mathrm{P}_{e}\left(x_{2, i}^{(j)}=1 \mid \bar{y}_{2, i}\right)}{\mathrm{P}_{e}\left(x_{2, i}^{(j)}=0 \mid \bar{y}_{2, i}\right)}\right) .
$$

\section{B. Unity-Rate Code}

Since the $(q, s)$ RLL encoder of Section III-A has a coding rate of $q / s$, it imposes an irrecoverable capacity loss. For the sake of circumventing this loss, we propose to invoke the recursive URC of [8] for scrambling. Explicitly, URC, having the generator polynomial $G(D)=\frac{1}{1+D}$, randomizes the input, hence resulting in an equal proportion of 1's and 0 's at the output without incurring any coding rate loss. However, unlike the $(q, s)$ RLL encoder, which strictly ensures a dimming value of $50 \%$ for each $s$-bit output symbol, the URC output may exhibit a fluctuating dimming value centered around $50 \%$. If these fluctuations are within the maximum flickering time period, which is around 5ms, they are imperceptible by the human eye because they are beyond its fusion frequency. Hence, they can be ignored.

For evaluating the impact of the fluctuations in dimming value, in Fig. 2 we plot the Probability Density Function (PDF) of the dimming value observed at the of the output of the memory-1 URC having the generator polynomial $G(D)=$ $\frac{1}{1+D}$. More specifically, we average the dimming value over $M$ bits, where $M=1000$ and $M=5000$ in Fig. 2. Practically, $M$ should meet the 'fusion-frequency' criterion, implying that if $D \mathrm{kbps}$ is the data rate, then we may average the dimming value over $M \leq 5 \times D$ bits, since any fluctuations within the block of $(5 \times D)$ bits are no longer perceptible by humans. We may observe in Fig. 2 that increasing the value of $M$ from 1000 to 5000 significantly reduces the deviation from the target dimming value of $50 \%$. Hence, considering the VLC systems, which may have data rates of several Megabits per second or even Gigabits per second, the fluctuations in the dimming value observed at the URC output would not result in flickering.

Alternatively, any potential flickering may also be eliminated by using compensation bits having the required value for adjusting the dimming level to $50 \%$. According to the PDF of Fig. 2a, the dimming value varies between $44 \%$ and $56 \%$. In the worst case, when the dimming value is $44 \%$, we are likely to have 4401 's in a block of 1000 bits. The resultant deviation from the target value of $50 \%$ may be adjusted by sending 120 additional 'On' bits, which would reduce the effective coding rate of the URC to 0.89 - albeit it would be still higher than the rate- $2 / 34 \mathrm{~B} 6 \mathrm{~B}$ as well as the rate- $1 / 2 \mathrm{BMC}$. Furthermore, the expected dimming value of the PDF of Fig. 2a is $49.6 \%$. Consequently, we only need 8 compensation bits on average, which implies an average URC coding rate of 0.992 .

\section{RESUlTS AND Discussions}

In this section, we use the 17-subcode IRCC of [6] as the outer component in the concatenated scheme of Fig. 1 for comparing the performance of the SISO RLL decoder of Section III-A and the URC of Section III-B to the BMCaided system of [5]. Explicitly, an IRCC is used, since it facilitates operating close to the maximum capacity achievable for a given inner code and the overall system throughput. This is achieved by optimizing the weighting coefficients of the subcodes of the IRCC using the optimization algorithm of [6] so that a narrow, but marginally open tunnel exits between the EXIT curves of the inner and outer decoders at the lowest possible SNR for a given system throughput. As a design example, let us consider a VLC system having an overall normalized throughput of $1 / 3$. The corresponding OOK AWGN capacity limit is $\mathrm{SNR}=-2.3 \mathrm{~dB}$ (or $E_{b} / N_{0}=2.5 \mathrm{~dB}$ ). The resultant EXIT curves of all the three schemes, namely of the BMCIRCC, 4B6B-IRCC and URC-IRCC arrangement, are plotted in Fig. 3. As depicted in Fig. 3, the convergence thresholds of the 4B6B-IRCC and URC-IRCC schemes are only $0.8 \mathrm{~dB}$ and $0.3 \mathrm{~dB}$ away from the capacity, respectively, while that of the BMC-IRCC design is $2.3 \mathrm{~dB}$ from the capacity. It may also be observed in Fig. 3 that the EXIT curve of the 4B6B inner code does not reach the $(1,1)$-point of perfect convergence to a vanishingly low BER owing to its non-recursive nature. Consequently, the two EXIT curves of the 4B6B-IRCC scheme crossover before reaching the $x=1$ line, which may result in a higher error floor. The corresponding BER performance is recorded in Fig. 4 for an interleaver length of both 3000 and 30,000 bits. In conformity with our EXIT chart predictions of Fig. 3, the URC-IRCC design significantly outperforms 


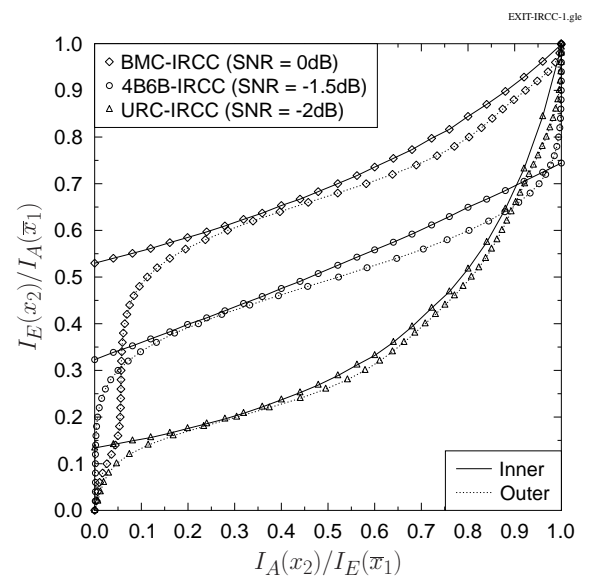

Fig. 3: EXIT curves of the concatenated inner-IRCC schemes, when the system throughput is fixed to $1 / 3$.

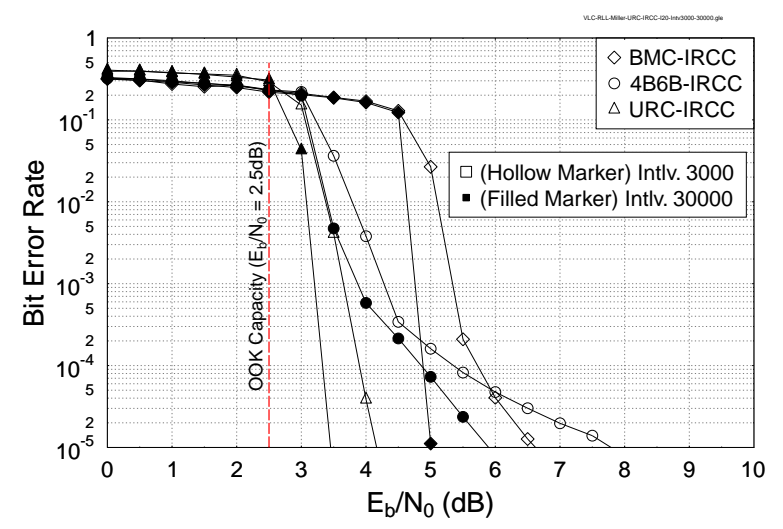

Fig. 4: Comparison of the achievable performance of the innerIRCC schemes, when 20 decoding iterations are invoked.

the 4B6B-IRCC and BMC-IRCC schemes. Furthermore, the 4B6B-IRCC arrangement exhibits turbo-cliff at a lower SNR compared to the BMC-IRCC, albeit this is achieved at the expense of a higher error floor.

We next compare the three inner components from the perspective of the achievable throughput in Fig. 5, where the OOK AWGN capacity is also plotted as a benchmarker. Explicitly, the 'maximum achievable rate' of Fig. 5 is computed on the basis of the area under the corresponding inner decoder's EXIT curve, while the 'achievable rate with IRCC' is obtained by optimizing the weighting coefficients of IRCC for the sake of maximizing the throughput at a given $\mathrm{SNR}^{2}$. Being a rate-1 code, URC is capable of achieving the OOK capacity, while the 4B6B and BMC schemes offer a significantly lower throughput, irrespective of the FEC code employed. Furthermore, as demonstrated in Fig. 5, the flexible nature of IRCC enables all the three coding schemes to approach the maximum achievable throughput. It is important to point out that the outer FEC code has to be carefully chosen so that the system operates close to the maximum achievable throughput. If the inner and outer

\footnotetext{
${ }^{2}$ For sake of avoiding a high error floor, it was ensured for the 4B6B-IRCC scheme that the inner and outer decoder's EXIT curves do not crossover before the $x=0.95$ line.
}

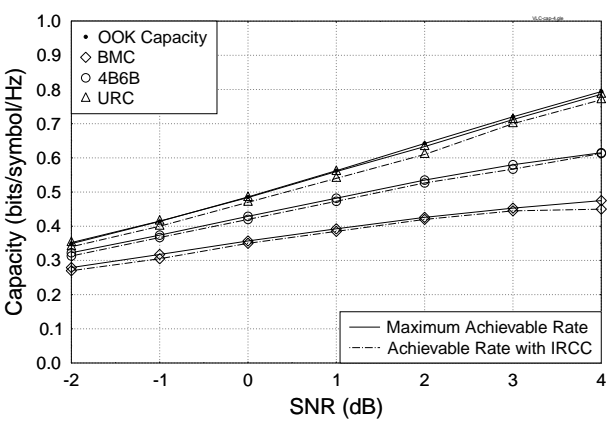

Fig. 5: Maximum throughput achievable with the various inner codes under consideration.

decoders' EXIT curves are not matched, the resultant EXIT chart may have a large open tunnel; hence the system would not operate close to the achievable capacity.

\section{COnclusions}

In this contribution, we conceived a SISO RLL decoder for facilitating the design of joint RLL-FEC coding schemes for VLC systems. As a further improvement, we also proposed a URC-aided VLC system, which is capable of maintaining a flicker-free dimming value of $50 \%$. We quantified the benefits of the proposed inner components by using them in conjunction with the IRCC and optimizing the designs using EXIT charts. The convergence thresholds of the proposed 4B6B-IRCC and URC-IRCC schemes are within $0.8 \mathrm{~dB}$ and $0.3 \mathrm{~dB}$ from the capacity, respectively, whereas that of the BMC-IRCC scheme is $2.3 \mathrm{~dB}$ from the capacity, when the system's normalized throughput is fixed to $1 / 3$. Furthermore, we demonstrate that the proposed schemes offer a higher throughput than the BMC-aided design.

\section{REFERENCES}

[1] S. Rajagopal, R. D. Roberts, and S. K. Lim, "IEEE 802.15.7 visible light communication: modulation schemes and dimming support," IEEE Communications Magazine, vol. 50, no. 3, pp. 72-82, March 2012.

[2] H. Wang and S. Kim, "New RLL decoding algorithm for multiple candidates in visible light communication," IEEE Photonics Technology Letters, vol. 27, no. 1, pp. 15-17, Jan 2015.

[3] — , "Soft-input soft-output run-length limited decoding for visible light communication," IEEE Photonics Technology Letters, vol. 28, no. 3, pp. 225-228, Feb 2016.

[4] _ "Bit-level soft run-length limited decoding algorithm for visible light communication," IEEE Photonics Technology Letters, vol. 28, no. 3, pp. 237-240, Feb 2016.

[5] X. Lu and J. L. Tiffany, "Achieving FEC and RLL for VLC: A concatenated convolutional-miller coding mechanism," IEEE Photonics Technology Letters, vol. 28, no. 9, pp. 1030-1033, May 2016.

[6] M. Tüchler, "Design of serially concatenated systems depending on the block length," IEEE Transactions on Communications, vol. 52, no. 2, pp. 209-218, February 2004.

[7] M. El-Hajjar and L. Hanzo, "EXIT charts for system design and analysis," IEEE Communications Surveys Tutorials, pp. 1-27, 2013.

[8] D. Divsalar, S. Dolinar and F. Pollara, "Serial concatenated trellis coded modulation with rate-1 inner code," in Proc. IEEE Global Telecommun. Conf., San Francisco, CA, Nov 2000, pp. 777-782. 